\title{
Remarks on the Cohen-Kaplan-Nelson bound
}

\author{
Tom Banks ${ }^{1}$ and Patrick Draper $\circledast^{2, *}$ \\ ${ }^{1}$ Department of Physics and NHETC, Rutgers University, Piscataway, New Jersey 08854, USA \\ ${ }^{2}$ Department of Physics, University of Illinois, Urbana, Illinois 61801, USA
}

(Received 26 February 2020; accepted 5 June 2020; published 16 June 2020)

\begin{abstract}
Cohen, Kaplan, and Nelson (CKN) conjectured that the UV and IR cutoffs of effective quantum field theories coupled to gravity are not independent, but are connected by the physics of black holes. We interpret the CKN bound as a scale-dependent depletion of the quantum field theory (QFT) density of states and discuss various aspects of the bound on small and large scales. For laboratory experiments, we argue that the bound provides small corrections to ordinary quantum field theory, which we estimate to be of order $m_{e} / M_{p}$ for $g-2$ of the electron. On large scales, we suggest a modification of the CKN bound due to the presence of cosmological horizons and discuss the connection with entropy bounds.
\end{abstract}

DOI: 10.1103/PhysRevD.101.126010

\section{INTRODUCTION}

Cohen, Kaplan, and Nelson [1] pointed out that UV and IR cutoffs on local effective quantum field theory might be correlated in the presence of gravity. Placing an ordinary EFT in a box of size $L$, CKN suggested that the UV cutoff $\Lambda_{\mathrm{UV}}$ should be low enough that states of characteristic energy density $\Lambda_{\mathrm{UV}}^{4}$ are not black holes: the Schwarzschild radius $r_{b}$ of the state should satisfy

$$
r_{b}\left(\Lambda_{\mathrm{UV}}, L\right)<L \Rightarrow \Lambda_{\mathrm{UV}}^{4} L^{3}<L M_{p}^{2}
$$

or

$$
\Lambda_{\mathrm{UV}}<\sqrt{M_{p} / L}
$$

up to order one numbers. This is an intrinsically gravitational cutoff that disappears in the limit that the Planck mass $M_{p}$ is taken to infinity.

It is obvious that EFT becomes suspect whenever gravitational backreaction is large enough to hide the box behind an event horizon. However, merely excluding "black hole states" from field theory has negligible impact on ordinary perturbative processes with small numbers of particles. ${ }^{1}$ The CKN bound is much stronger than this.

\footnotetext{
*pdraper@illinois.edu

${ }^{1}$ Apart from rendering the perturbative series somewhat better defined. States of energy $M$ in regions of size $R$ with entropy $S>M R$ are problematic because $e^{S} e^{-M R}>1$. The Bekenstein bound excludes these states, but the CKN bound is even stronger, amounting to $S_{\mathrm{EFT}}<R^{3 / 2}$, where $S_{\mathrm{EFT}}$ is the entropy that can be well-described by quantum field degrees of freedom.

Published by the American Physical Society under the terms of the Creative Commons Attribution 4.0 International license. Further distribution of this work must maintain attribution to the author(s) and the published article's title, journal citation, and DOI. Funded by SCOAP ${ }^{3}$.
}

We will give a new interpretation of the bound as a depletion of the EFT density of states. This interpretation gives a general definition of the "IR cutoff" in the bound, and provides a method to estimate the order of magnitude of CKN effects on particle physics processes.

We also advocate for a more general bound [2], applicable to the use of EFT in any space-time, which is independent both of coordinates and processes. This is the bound

$$
S_{\mathrm{EFT}}<c A^{\frac{d-1}{d}},
$$

where $A$ is the area of the maximal $d-2$ volume of a spacelike slice in a null foliation of any causal diamond in the space-time and $S_{\mathrm{EFT}}$ is an entropy, counting degrees of freedom that can be well described by field theory. More precisely, $S_{\mathrm{EFT}}$ is to be interpreted as the logarithm of the dimension of the gauge invariant Hilbert space of the field theory in the causal diamond. This bound, like that of CKN, determines a UV cutoff of field theory that is correlated with a measure of the size of the region in which EFT is used. However, it is phrased in terms of quantities that are independent of particular processes and are geometric invariants. $c$ is an $\mathcal{O}(1)$ number that is independent of the diamond. ${ }^{2}$ This bound is satisfied in a large collection of models that are well-defined, unitary, and causal, as well as consistent with asymptotic invariance under spacetime translation and spatial rotation symmetries on the conformal boundary of Minkowski space [3-6]. Of course, ultimately the covariant bound has to be interpreted in particular

\footnotetext{
${ }^{2}$ It is plausible that it is also independent of the particular EFT under consideration. Arguments involving black hole formation are always subject to the caveat that we do not know, even in classical general relativity, exactly which initial scattering states lead to black holes.
} 
coordinates: we have to choose a time slicing in order to have a Hamiltonian, Hilbert space formulation of the EFT. In the rest of this paper we will always work in the most symmetric static coordinate system available.

Ordinarily, in a large box of volume $V=L^{3}$, the number of states in a momentum space volume $d^{3} p$ is

$$
d N \sim V d^{3} p .
$$

This leads, for example, to the "quartic divergence in the vacuum energy density,"

$$
\Delta E / V \sim V^{-1} \int d \epsilon \epsilon D(\epsilon), \quad D(\epsilon) \sim V \epsilon^{2}
$$

summing over zero-point energies of the single-particle states. We will interpret the CKN bound as a conjecture that the one-particle density of states that is well-described by quantum field theory is depleted in a scale-dependent way, as though at energy $\epsilon$ the modes are confined to a box of size $L(\epsilon)<M_{p} / \epsilon^{2}$, or

$$
d N \sim M_{p}^{3} \epsilon^{-6} d^{3} p
$$

saturating the inequality.

In an expanding universe of Hubble parameter $H$, the "bare vacuum energy density" is of order $H^{2} M_{p}^{2}$. Modes of energy lower than $\sqrt{H M_{p}}$ appear to give a quantum correction to the vacuum energy density which is of order the bare value. Modes of energy $\epsilon>\sqrt{H M_{p}}$ contribute to the usual fine-tuning problem. It is these modes that are depleted by the CKN bound, since $L(\epsilon)<H^{-1}$, and it was suggested in [1] that the low UV cutoff on ordinary QFT might explain why the cosmological constant is not quartically sensitive to the highest energy scales through radiative corrections. With the QFT density of states (5) we can estimate that high energy modes contribute

$$
\begin{aligned}
\Delta E / V & \sim H^{3} \int_{\sqrt{H M_{p}}}^{\infty} d \epsilon M_{p}^{3} \epsilon^{-3} \\
& \sim H^{2} M_{p}^{2} .
\end{aligned}
$$

Although this result does not explain the large size of the universe, it might resolve the fine-tuning problem. AdS/CFT arguments support a similar conclusion [7].

The goal of this paper is twofold. First, since CKN used Schwarzschild black hole arguments to draw conclusions about the cosmological constant, it is interesting to ask how the conclusions are modified when Schwarzschild-de Sitter (SdS) black holes are used instead. We investigate this question in Secs. II and III. The modifications we suggest imply that on cosmological scales the QFT degrees of freedom are depleted even more rapidly than (5); however, the CKN bound interpreted as an entropy bound is qualitatively unchanged. Second, we revisit the implications of the CKN bound for particle physics. It is not immediately clear how to impose (2): which $L$ should be used? What is the impact on IR-dominated processes, where typical momenta are not of order $\Lambda_{\mathrm{UV}}$ ? We argue that the depleted density of states gives a processindependent prescription for estimating at least the order of magnitude of CKN effects. In Sec. IV we apply this prescription to $g-2$ and find that the size of the effects estimated this way is much smaller than the estimate in [1]. In Sec. $\mathrm{V}$ we conclude with brief comments about the relevance of the CKN bound to scalar potentials, the equivalence principle, and dS thermodynamics.

\section{MODIFICATION ON COSMOLOGICAL SCALES}

The CKN bound as stated above requires some modification on cosmological scales. (In this section we set $M_{p}=1$.) Exciting finite-energy states over large scales changes the cosmological horizon. For example, in de Sitter space with dS length $\ell$, there is a cosmological horizon at $r_{c}=\ell$ in static coordinates. Therefore one might like to consider EFT in boxes as large as $L=r_{c}$ and place a CKNtype bound on the UV cutoff. However, there are no black holes in this asymptotic spacetime with radius larger than the Nariai size $r_{N}=\ell / \sqrt{3}$.

To resolve this puzzle, we can extend the CKN bound to

$$
r_{b}\left(\Lambda_{\mathrm{UV}}, L ; \ell\right)<L<r_{c}\left(\Lambda_{\mathrm{UV}}, L ; \ell\right) .
$$

Here $r_{b}$ and $r_{c}$ are the black hole and cosmological horizons of the $\mathrm{SdS}$ spacetime with $\mathrm{dS}$ length $\ell$ and mass $m \equiv \Lambda_{\mathrm{UV}}^{4} L^{3}$. The $\mathrm{SdS}$ horizons are two of the three solutions to $1-\frac{2 m}{r}-\frac{r^{2}}{\ell^{2}}=0$. Therefore, both conditions (7) can be imposed at once by requiring

$$
1-\frac{2 m}{L}-\frac{L^{2}}{\ell^{2}}>0
$$

which leads to

$$
\Lambda_{\mathrm{UV}}<\left(\frac{1}{2 L^{2}}-\frac{1}{2 \ell^{2}}\right)^{\frac{1}{4}} .
$$

The bound (9) reduces to that of $\mathrm{CKN}$ for $L \ll r_{N}$. On larger scales, it encodes the requirement that EFT should not include states for which the cosmological horizon is inside the box. In particular, for $L \rightarrow \ell$, the UV cutoff goes to zero.

We can give two interpretations of this result. We might treat the bound (9) as an effective modification of the density of states, as above. In this case we again obtain a zero-point contribution of order the bare scale, $\Delta E / V \sim 1 / \ell^{2}$. On the other hand, the vanishing UV cutoff 
may instead imply that there is no renormalization of the cc from conventional EFT. This is consistent with substantial evidence accumulated by Banks and Fischler that the cc should be thought of as a fixed parameter [8]. A similar conclusion was recently drawn by Bramante and Gould [9]. We will mainly suggest an interpretation of (9) in relation to entropy bounds.

\section{RELATION TO ENTROPY BOUNDS}

It was argued in [2] that the CKN bound arises from a form of the covariant entropy principle $[10,11]$ applied to field-theoretic degrees of freedom,

$$
S_{\diamond, \mathrm{EFT}}<c A_{\diamond}^{\frac{3}{4}},
$$

where $\diamond$ denotes a causal diamond, $S_{\diamond, \mathrm{EFT}}$ is the entropy associated with degrees of freedom that can be wellapproximated by local EFT inside the diamond, and $A_{\diamond}$ is the area of the holographic screen, the maximal-area 2 -surface on the boundary of the diamond. $c$ is a number of order one. For small causal diamonds, the entropy in local QFT degrees of freedom is of order $\left(L \Lambda_{\mathrm{UV}}\right)^{3}$, so replacing $A_{\diamond} \rightarrow L^{2}$, we obtain

$$
\left(L \Lambda_{\mathrm{UV}}\right)^{3} \lesssim\left(L^{2}\right)^{\frac{3}{4}}
$$

recovering the CKN bound (2). Our de Sitter space result can also be viewed as an application of the general bound on EFT entropy to a particular space-time and a particular coordinate system.

More generally, in the static patch, the entropy in states that can be well described by local QFT degrees of freedom (at least over times of order $\ell$ ) increases with the box size as

$$
d S_{\mathrm{EFT}} \simeq\left(\Lambda_{\mathrm{UV}}(L)\right)^{3} L^{2} d L .
$$

If the UV cutoff on states of characteristic size $L$ does not fall too quickly with $L$, we recover $S_{\mathrm{EFT}} \simeq\left(L \Lambda_{\mathrm{UV}}(L)\right)^{3}$. This is the case for the ordinary CKN bound (2). However, if $\Lambda_{\mathrm{UV}}(L)$ is taken to saturate (9), the estimate $S_{\mathrm{EFT}} \simeq$ $\left(L \Lambda_{\mathrm{UV}}(L)\right)^{3}$ is invalid for $L$ of cosmological size. Indeed, it predicts $S_{\mathrm{EFT}} \rightarrow 0$. Instead, (12) indicates that $d S_{\mathrm{EFT}} / d L$ is peaked at $L=\ell / 2$ and goes to zero as $L \rightarrow \ell \cdot d S_{\mathrm{EFT}} / d L$ is shown in Fig. 1.

In other words, in the largest causal diamonds, most of the entropy that can be well described by local QFT degrees of freedom over times of order $\ell$ corresponds to states that are localized an $\mathcal{O}(1)$ distance inside the diamond, rather than roughly homogeneous and horizon-sized. This is what we learn about field theory entropy from (9) and is consistent with the arguments of [12].

Integrating Eq. (12) and replacing $L^{2} \rightarrow A_{\diamond}$, we find

$$
S_{\diamond, \mathrm{EFT}}<f(L / \ell) A_{\diamond}^{\frac{3}{4}},
$$

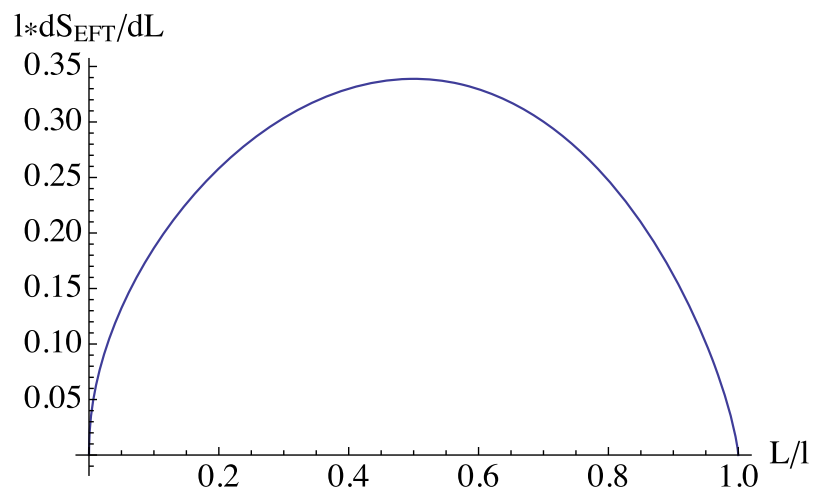

FIG. 1. Most of the states that can be well-described by QFT are localized well inside the cosmological horizon.

where $f$ is a function that always of order one for $L$ between zero and $\ell$. We find that the modified CKN bound (9) still qualitatively saturates the $S<A^{\frac{3}{4}}$ bound on field theory entropy.

\section{CKN AND PARTICLE PHYSICS}

$\mathrm{CKN}$ argued that their bound is conceivably testable in precision measurements, and they estimate the effects of correlated UV and IR cutoffs on the one-loop contribution to $g-2$ of the electron,

$$
\delta(g-2) \sim \frac{\alpha}{\pi}\left[\left(\frac{m_{e}}{\Lambda_{\mathrm{UV}}}\right)^{2}+\left(\frac{1}{m_{e} L\left(\Lambda_{\mathrm{UV}}\right)}\right)^{2}\right]
$$

with $L$ and $\Lambda$ related by Eq. (2). (See also the recent work [13].) The estimate (14) comes from placing hard momentum cutoffs on the usual Feynman integral and correlating them as in (2). However, it is not clear that this is a physically sensible way to estimate the effects of the bound.

An advantage of the density of states (5) is it provides a conservative, process-independent method to estimate the impact of CKN physics on QFT radiative corrections. For UV-finite processes with a single characteristic energy scale $E$, the physical interpretation of (5) is relatively simple: the standard QFT description is modified as though the field theory was placed in a finite volume $(L(E))^{3}$. The finite volume depletes the density of states in the correct way around the most relevant modes. Below we demonstrate the application of this method to $g-2$, finding that the impact of the CKN-depleted density of states is much smaller than Eq. (5) suggests.

Before we do so, however, we emphasize that this prescription differs from an exact implementation of (5): it will underestimate the depletion of states with energies $\gg E$ and overestimates it for states with energies $\ll E$. These errors can be neglected as long as the Feynman integrands are sufficiently peaked around $E$ that the CKN corrections are also dominated by the depletion around $E$. For $g-2$ we will check this explicitly for modes of energy 
$\Lambda \gg E$ : depleting these modes by the more appropriate box size $L(\Lambda)$ corrects $g-2$ by an amount parametrically smaller than the depletion of the dominant modes around E. Also, although Eq. (5) appears strongly infraredsensitive, it is actually reflecting the fact that IR physics is very insensitive to the depletion: the box size is growing faster than the scale $1 / \epsilon$ for low $\epsilon$. Thus low energy physics approaches the ordinary infinite volume behavior.

Physically, since the effective box size is much larger than $1 / E$ for all energies $E$ satisfying $H \ll E \ll M_{p}$ (where $H$ is the Hubble scale), the effects are small at all such energies. For example, $g-2$ is a process with characteristic energy $m_{e}$. The effective finite volume is the size of the sun. Since the momentum support of the Feynman integrand is also of order $m_{e}$, the box size varies by $\mathrm{O}(1)$ over this region of support. Therefore, we only expect our prescription to work at the $\mathrm{O}(1)$ level.

With these caveats, we proceed to the estimate. The usual one-loop correction to $g-2$ is schematically

$$
\begin{aligned}
(g-2) \sim & e^{2} m_{e}^{2} \int_{0}^{1} d x d y d z \delta(x+y+z-1) \\
& \times \int \frac{d \ell_{0}}{2 \pi} \int \frac{d^{3} \ell}{(2 \pi)^{3}} \frac{z(1-z)}{\left(\ell^{2}+\Delta^{2}\right)^{3}}, \\
\Delta^{2} \equiv & (1-z)^{2} m_{e}^{2}
\end{aligned}
$$

(in Euclidean space, at leading order in the momentum of the external photon, and omitting order-one numbers). The integral is dominated by loop momenta of order $m_{e}$, so a more appropriate estimate of the effects of the scaledependent density of states is to place the system in a box of size $L\left(m_{e}\right)=M_{p} / m_{e}^{2}$. (This scale is enormous, and so the rest of the exercise is academic, showing only that such corrections are negligibly small.) Finite volume effects can then be estimated with standard techniques (see, e.g., [14]). We use an exponential representation of the propagators and discretize spatial momenta,

$$
\begin{aligned}
\frac{1}{\left(\ell^{2}+\Delta\right)^{3}} & \rightarrow \int d \rho \rho^{2} e^{-\rho\left(\ell_{0}^{2}+\Delta\right)} e^{-\rho \ell_{i}^{2}} \ell_{i} \\
& \rightarrow \frac{2 \pi \nu_{i}}{L} \int d^{3} \ell_{i} \rightarrow \frac{1}{L^{3}} \sum_{\nu_{i}} .
\end{aligned}
$$

Each of the spatial momentum sums can be reorganized with Poisson summation,

$$
\sum_{\nu} e^{-\rho(2 \pi \nu / L)^{2}} \rightarrow \frac{L}{\sqrt{\rho}} \sum_{n} e^{-\frac{1}{\rho}\left(\frac{L n}{4 \pi}\right)^{2}}
$$

again dropping order one numbers. For large $L$ the sum is dominated by $n=0, \pm 1$, where $n=0$ corresponds to the infinite volume limit. We can then perform the integrals over $\ell_{0}$ and the Feynman parameters to obtain

$$
\begin{aligned}
\delta(g-2)_{\mathrm{IR}} & \left.\equiv(g-2)\right|_{L m_{e} \gg 1}-\left.(g-2)\right|_{L \rightarrow \infty} \\
& \sim \frac{\alpha}{\pi} \int d \rho\left(\frac{e^{-\frac{L^{2}}{16 \pi^{2} \rho}}}{m_{e} \rho^{3 / 2}}+\mathcal{O}\left(\frac{1}{m_{e} \sqrt{\rho}}\right)\right) .
\end{aligned}
$$

Since the integral is dominated by $\rho \sim L^{2}$, higher-order terms in $1 /\left(\sqrt{\rho} m_{e}\right)$ can be dropped. Performing the $\rho$ integral we obtain the leading correction to $g-2$ from a finite box of size $L\left(m_{e}\right)$,

$$
\delta(g-2)_{\mathrm{IR}} \sim \frac{1}{m_{e} L\left(m_{e}\right)} \sim \frac{m_{e}}{M_{p}} .
$$

Another way to arrive at this result is to note that the modification to $g-2$ is the difference between doing an ordinary integral in a momentum range around $m_{e}$ and approximating the integral with a Riemann sum with momentum bins of size $2 \pi / L$. The "error" in the Riemann sum approximation to this region of the integral is linear in $1 / L$.

Including also an ordinary UV cutoff (without CKN effects) for comparison, we obtain

$$
\delta(g-2) \sim \frac{\alpha}{\pi}\left[\left(\frac{m_{e}}{\Lambda_{\mathrm{UV}}}\right)^{2}+\left(\frac{m_{e}}{M_{p}}\right)\right] .
$$

We see that the depleted density of states gives results comparable to ordinary UV contributions for $\Lambda \gtrsim$ $\sqrt{m_{e} M_{p}} \sim 10^{7} \mathrm{GeV}$.

We can obtain a similar estimate from the effect of the density of states on corrections to $g-2$ from new physics at $\Lambda_{\mathrm{UV}}$ by putting this part of the momentum integral in a box of size $L\left(\Lambda_{\mathrm{UV}}\right)$,

$$
\begin{aligned}
\delta(g-2)_{\mathrm{UV}} & \sim \frac{\alpha}{\pi}\left[\left(\frac{m_{e}}{\Lambda_{\mathrm{UV}}}\right)^{2}\left(1+\frac{1}{\Lambda_{\mathrm{UV}} L\left(\Lambda_{\mathrm{UV}}\right)}\right)\right] \\
& \sim \frac{\alpha}{\pi}\left[\left(\frac{m_{e}}{\Lambda_{\mathrm{UV}}}\right)^{2}+\frac{m_{e}^{2}}{M_{p} \Lambda_{\mathrm{UV}}}\right] .
\end{aligned}
$$

The first term is the typical contribution from a standard UV cutoff, ignoring CKN corrections, and the second is the typical CKN correction to this contribution as estimated above. We see that the second term is of higher order compared to the second term in Eq. (20); most of the modifications from a $\mathrm{CKN}$-type bound indeed arise from momentum scales around $m_{e}$.

It is also possible to improve on this estimate in a systematic way by depleting the modes according to Eq. (5) at all scales. This requires some commitment to how the modes are pruned. A more detailed analysis will be presented elsewhere; here we simply sketch the idea. We can, for example, take a cubic lattice with points labeled by an integer 3 -vector $\vec{n}$, and relate $\vec{n}$ to momentum states via $d n_{i} \sim L\left(E_{p}\right) d p_{i}$. Then we can define modified field operators as 


$$
\phi(x) \rightarrow \sum_{n} L(|p|)^{-3} \frac{1}{\sqrt{2|p|}}\left(a_{p} e^{i p \cdot x}+a_{p}^{\dagger} e^{-i p \cdot x}\right),
$$

taking a massless scalar for simplicity. Here $\vec{p}$ is implicitly a function of $\vec{n}$. If we impose $\left[a_{p}, a_{p^{\prime}}^{\dagger}\right]=\delta_{n, n^{\prime}} L(|p|)^{3}$, we can compute, for example, the real-space correlation function $D(x)=\langle 0|\phi(x) \phi(0)| 0\rangle$. One finds that the usual result of order $1 /|x|^{2}$ is corrected to $1 /|x|^{2}\left(1+\mathcal{O}\left(1 / x M_{p}\right)\right)$. This is consistent with our estimate above: a process dominated by a momentum scale of order $p$ will be corrected at order $p / M_{p}$.

\section{DISCUSSION}

We have discussed an interpretation of the CKN bound as a depletion of the QFT density of states, and argued that the usual Schwarzschild black hole argument requires modification on cosmological scales. This modification supports the idea that most field theory states in dS space are localized well inside the cosmological horizon. We have also estimated the effect of the CKN bound on small scale physics and found that it is negligibly small.

Let us conclude with several additional comments.

(i) The impact of the CKN bound on scalar fields is of interest. The density of states (5) implies that the effective potential for scalar fields computed in ordinary quantum field theory [e.g., the field dependent terms in the vacuum energy (6)] should not be thought of as providing a correction to the cosmological constant.

On the other hand, as our analysis of $g-2$ indicates, the predictions of quantum field theory for local measurements at SM energies are not substantially modified by the CKN bound. In light of this, the effective potential computed in ordinary QFT without the density of states (5) should accurately capture loop corrections to on-shell scalar processes with loop momenta $k$ and external momenta $k_{\text {ext }}$ satisfying

$$
L(k)^{-1} \ll k_{\mathrm{ext}} \ll k .
$$

The first inequality is a CKN bound and the second from the interpretation of the effective potential as the leading term in a derivative expansion. Thus the usual quadratic divergence in scalar masses is still a problem. The extent of the electroweak hierarchy problem that can be inferred from ordinary QFT is characterized by the UV scale

$$
\Lambda \sim \sqrt{m_{W} M_{p}},
$$

at which $L$ becomes shorter than the weak scale and the Higgs no longer fits into the CKN box.
We are quite uncertain about the extent to which EFT calculations of an effective potential correspond to any calculation in a true theory of quantum gravity. String theory teaches us to extract effective Lagrangians from scattering amplitudes. Sen et. al. and Seiberg [15-17] have shown how to extract oneloop on-shell mass renormalization in this manner, while Kaplunovsky has done the same for gauge coupling renormalization [18]. Our remarks above about on-shell scalar mass renormalization are thus on pretty safe grounds. Interpretation of the effective potential as a calculable contribution to the cc, however, is quite problematic, as illustrated by the $\mathrm{CKN}$ bound and reinforced by a host of other more rigorous evidence [8].

(ii) It is known from tests of the equivalence principle that "vacuum loops gravitate" [19]. In brief, the idea is that radiative corrections to the binding energies of different nuclei $A$ and $B$ are much larger than the precision with which $m_{\text {inertial }}^{A} /$ $m_{\text {gravitational }}^{A}=m_{\text {inertial }}^{B} / m_{\text {gravitational }}^{B}$ has been tested. Therefore, at least in the background fields of a nucleus, loops couple to gravity [19]. This is not inconsistent with the CKN bound, because the relevant length scales in the radiative contributions to nuclear binding energies are $\mathcal{O}(\mathrm{fm})$. $\mathrm{CKN}$ effects are expected to be of order $\mathrm{GeV} / M_{p}$.

(iii) The thermodynamics of SdS black holes indicates that general localized excitations in dS space are low-entropy constrained states of the dS thermal bath (see $[12,20]$ and references therein; also more recently [21-23].) Most of these states are black holes that do not have a field-theoretic description. In any case, the fact that localized states have low entropy indicates that they are unstable over sufficiently long times. All "field theory states" are localized and the bounds above should be understood to apply on timescales shorter than the time over which they decay back into the dS bath.

(iv) Finally, the ideas in this paper should certainly have implications for field theory models of inflation and inflationary fluctuations, but the authors do not yet understand what they are. We hope to return to this question in future work.

It would be interesting to study further the implications of these sorts of bounds for other precision tests and scalar field cosmology.

\section{ACKNOWLEDGEMENTS}

The work of P. D. is supported by NSF Grant No. PHY1719642. The work of T. B. is partially supported by the U.S. Department of Energy under Grant No. DESC0010008. 
[1] A. G. Cohen, D. B. Kaplan, and A. E. Nelson, Effective Field Theory, Black Holes, and the Cosmological Constant, Phys. Rev. Lett. 82, 4971 (1999).

[2] T. Banks, On the limits of effective quantum field theory: Eternal inflation, landscapes, and other mythical beasts, arXiv:1910.12817.

[3] T. Banks, W. Fischler, S. Kundu, and J. F. Pedraza, Holographic space-time and black holes: Mirages as alternate reality, arXiv:1401.3341.

[4] T. Banks, Current Algebra on the conformal boundary, and the variables of quantum gravity, arXiv:1511.01147.

[5] T. Banks, Note on localized objects as constrained states of holographic variables, arXiv:1710.01211.

[6] T. Banks, Holographic space-time and quantum information, Front. Phys. 8, 111 (2020).

[7] S. D. Thomas, Holography Stabilizes the Vacuum Energy, Phys. Rev. Lett. 89, 081301 (2002).

[8] T. Banks and W. Fischler, Why the cosmological constant is a boundary condition, arXiv:1811.00130.

[9] J. Bramante and E. Gould, Material matter effects in gravitational UV/IR mixing, Phys. Rev. D 101, 084022 (2020).

[10] W. Fischler and L. Susskind, Holography and cosmology, arXiv:hep-th/9806039.

[11] R. Bousso, The Holographic principle for general backgrounds, Classical Quantum Gravity 17, 997 (2000).

[12] T. Banks, TASI Lectures on holographic space-time, SUSY and gravitational effective field theory, in Proceedings, Theoretical Advanced Study Institute in Elementary Particle Physics (TASI 2010). String Theory and Its Applications: From meV to the Planck Scale: Boulder, Colorado, USA (World Scientific, Singapore, 2010).
[13] J. Bramante and E. Gould, Anomalous anomalies from virtual black holes, Phys. Rev. D 101, 055007 (2020).

[14] A. S. Kronfeld, Uses of effective field theory in lattice QCD, in At the Frontiers of Particle Physics, Handbook of QCD (World Scientific, Singapore, 2002), Chap. 39, Vol. 4, pp. 2411-2477.

[15] N. Seiberg, Anomalous dimensions and mass renormalization in string theory, Phys. Lett. B 187, 56 (1987).

[16] R. Pius, A. Rudra, and A. Sen, Mass renormalization in string theory: Special states, J. High Energy Phys. 07 (2014) 058.

[17] R. Pius, A. Rudra, and A. Sen, Mass renormalization in string theory: General states, J. Hisgh Energy Phys. 07 (2014) 062.

[18] V.S. Kaplunovsky, One loop threshold effects in string unification, Nucl. Phys. B307, 145 (1988); Erratum, Nucl. Phys. B382, 436 (1992).

[19] J. Polchinski, The cosmological constant and the string landscape, in The Quantum Structure of Space and Time: Proceedings of the 23rd Solvay Conference on Physics. Brussels, Belgium, 2005 (World Scientific, Singapore, 2006), pp. 216-236.

[20] T. Banks, B. Fiol, and A. Morisse, Towards a quantum theory of de Sitter space, J. High Energy Phys. 12 (2006) 004.

[21] C. V. Johnson, Specific heats, and Schottky peaks for black holes in extended thermodynamics, Classical Quantum Gravity 37, 054003 (2020).

[22] J. Dinsmore, P. Draper, D. Kastor, Y. Qiu, and J. Traschen, Schottky anomaly of deSitter black holes, Classical Quantum Gravity 37, 054001 (2020).

[23] C. V. Johnson, de Sitter black holes, Schottky peaks, and continuous heat engines, arXiv:1907.05883. 